\title{
Cardiac manifestations of tuberculosis in a tertiary care center of Nepal
}

\author{
Smriti Shakya, Sunil Chandra Jha
}

Department of Cardiology, Manmohan Cardiothoracic Vascular and Transplant Center, Maharajgunj, Nepal.

Corresponding Author: Smriti Shakya

Department of Cardiology, Manmohan Cardiothoracic Vascular and Transplant Center, Maharajgunj, Nepal.

Email: drsmritishakya@gmail.com

\section{Abstract}

Background and Aims: Tuberculosis remains an important etiological cause of pericarditis and pericardial effusion in developing countries like Nepal. The objective of this study is to identify the various presentations of tuberculous pericarditis along with the demographic profile in our context and their short term outcome.

\section{Methods:}

We studied 53 patients from September 2015 to August 2017 regardless of age and gender who presented to Manmohan Cardiothoracic Vascular and Transplant Center with pericarditis of tubercular origin. The various manifestations of the disease were categorized with 2D echocardiography. Pericardiocentesis was done in patients with large pericardial effusion especially in cardiac tamponade and pericardiectomy done in chronic constrictive pericarditis(CCP). Antitubercular therapy with steroids was instituted.

Results:

Out of 53 patients, $62 \%$ were male and $38 \%$ were female. The ages ranged from $6-71$ years $(42 \pm 19.5)$. Twenty three percent of patients were from the age group 61-70yrs, $20 \%$ seen in age group 21-30 years, $8 \%$ in less than 10 yrs and $2 \%$ in above $70 y$ rs old patients. The most common manifestation seen was large pericardial effusion (32\%), followed by CCP $(22.6 \%), 19 \%$ presented in cardiac tamponade, $2 \%$ had pyopericardium, $2 \%$ had perimyocarditis and $4 \%$ had acute pericarditis. Adenosine deaminase (ADA) was positive in $75 \%$ of the cases when pericardial fluid was tapped. Two patients developed CCP during follow up. Two patients succumbed to death during hospital stay.

Conclusion:

A high index of suspicion of tubercular pericarditis is inevitable in our settings where other sophisticated investigations are still lacking.

Key words: Cardiac tamponade, Pericardial effusion, Tuberculosis.

DOI: http://dx.doi.org/10.3126/njh.v15i1.19714

\section{Introduction}

The World Health Organization (WHO) estimates that each year more than 8 million new cases of tuberculosis occur and approximately 3 million persons die from it, most occurring in developing countries due to homelessness, malnourishment, and over crowdedness, and where human immunodeficiency virus (HIV) infection may be common. ${ }^{1}$ However, there has been a significant decline in tuberculosis in industrialized countries over the past 100 years. $^{2}$

Tuberculous Pericarditis, one of the manifestation of Mycobacterium tuberculosis infection, is found in approximately $1 \%$ of all autopsied cases of tuberculosis and in $1 \%$ to $2 \%$ of instances of pulmonary TB. ${ }^{3}$ Tuberculous pericarditis presents clinically in 3 forms i.e. pericardial effusion, constrictive pericarditis, and a combination of effusion and constriction. ${ }^{3}$ A "definite" diagnosis of tuberculous pericarditis in tuberculosis endemic countries is based on the demonstration of tubercle bacilli in pericardial fluid or on a histological section of the pericardium; "probable" tuberculous pericarditis is based on the proof of tuberculosis elsewhere in a patient with otherwise unexplained pericarditis, a lymphocytic pericardial exudate with raised adenosine deaminase(ADA) levels, and/or appropriate response to a trial of antituberculosis chemotherapy. ${ }^{3}$

Tuberculosis (TB) is responsible for about $4 \%$ of cases of acute pericarditis, $7 \%$ of cases of cardiac tamponade and about $6 \%$ of cases of constrictive pericarditis in the developed countries however in the underdeveloped countries, TB is the leading cause of pericarditis. ${ }^{4}$ It leads to a mortality of $17 \%$ to $40 \%$ so that early diagnosis and institution of appropriate therapy are critical to prevent mortality. ${ }^{3}$ Despite prompt anti tubercular treatment and use of corticosteroids, constrictive pericarditis is one of the most serious sequelae of TB pericarditis and occurs in $30 \%$ to $60 \%$ of these patients. Tuberculosis as an etiology of constrictive pericarditis is more common than other causes in Africa and Asia. ${ }^{5}$

\section{Methodology:}

All the patients who presented to Manmohan Cardiothoracic Vascular and transplant center and were diagnosed to have pericardial effusion (small/ moderate/ large

@Nepalese Heart Journal. Nepalese Heart Journal retains copyright and work is simultaneously licensed under Creative Commons Attribution License CC - BY 4.0 that allows others to share the work with an acknowledgement of the work's authorship and initial publication in this journal. 
whether with or without tamponade) or chronic constrictive pericarditis of tubercular origin between September 2015 and August 2017 were included. Patients of any age or gender were taken. Detailed history about fever, shortness of breath, orthopnea, cough, chest pain, leg swelling or abdominal distension was inquired with clinical evaluation such as jugular venous distension, pericardial rub or distant heart sounds were mentioned. ECG, chest $x$-ray, 2D echocardiography (GE, Vivid 7) along with baseline blood investigations were sent.

The size of pericardial effusion was measured during diastole. The pericardial effusion was classified as small $(<10 \mathrm{~mm})$, moderate $(10-20 \mathrm{~mm})$ and large $(>20 \mathrm{~mm})$. The cardiac tamponade was diagnosed by presence of large pericardial effusion with swinging heart, diastolic collapse of right atrium and right ventricle, variations in $\mathrm{E}$ velocities during respiration across the mitral valve, tricuspid valve and pulmonary outflow that are greater than 25, 50 and $30 \%$ and Inferior Venacava (IVC) plethora (dilatation $>20 \mathrm{~mm}$ and $<50 \%$ reduction in diameter of IVC with respiratory phases). The constrictive pericarditis was diagnosed by biatrial enlargement, ventricular septal shift/ shudder, prominent diastolic flow reversals in hepatic vein in expiration, preserved or elevated e', annulus reversus, annulus paradoxus, respiratory variation in mitral inflow velocity, plethora of IVC, pericardial thickening, distortion of ventricular contour, pericardial effusion if effusive-constrictive pericarditis. Chronic constrictive pericarditis was confirmed by CECT chest showing thickened and calcified pericardium as well as dilated suprahepatic inferior venacava.

Those patients who were in cardiac tamponade or large pericardial effusion whose diagnosis was in dilemma underwent pericardiocentesis under fluoroscopic guidance or pericardial window made (if pericardiocentesis was not feasible). Some patients had pericardial biopsy when pericardial window was made. The pericardial fluid was sent for cytology (total white cell count, differential count), biochemistry [protein, lactate dehydrogenase (LDH), Adenosine deaminase (ADA), sugar], microbiology [Acid Fast Bacilli (AFB) stain, culture, gram stain] as well as for malignant cell cytology. In addition, patients underwent tests for HIV, Hepatitis B and C, blood biochemistry, Rheumatoid factor (RF), Anti Nuclear Antibody (ANA), Thyroid Function Test (TFT), Erythrocyte Sedimentation Rate (ESR), C-reactive protein (CRP), sputum for AFB stain, gram stain and culture. The cut-off point for ADA of pericardial fluid and pleural fluid to be positive was taken as $\geq 40 \mathrm{U} / \mathrm{L}$ for diagnosis of tuberculosis. Tuberculin Skin Test was also done and categorized as per Centers for Disease Control and prevention. An induration of 5 or more millimeters is considered positive in -HIV-infected persons, a recent contact of a person with TB disease, persons with fibrotic changes on chest radiograph consistent with prior TB, persons who are immunosuppressed, 10 or more millimeters considered positive in recent immigrants $(<5$ years $)$ from high-prevalence countries, injection drug users, children $<4$ years of age, infants, children, and adolescents exposed to adults in high-risk categories and an induration of 15 or more millimeters is considered positive in any person with no known risk factors for TB.

When the effusion was small to moderate, the diagnosis of tuberculosis was supported by finding the tubercular lesion elsewhere in the body or other supportive investigations for example pleural fluid analysis instead of pericardial fluid analysis if they had coexisting pleural effusion where the cutoff value of ADA was taken $\geq 40 \mathrm{U} / \mathrm{L}$ to diagnose tubercular pleural effusion. When the diagnostic dilemma occurred, a trial of anti tubercular drugs along with steroids were given and the response was observed by regular follow up of the patient until resolved after which they were enrolled in the study. Those patients were excluded whose effusion didn't resolve after the course of anti-tubercular medications. All those patients with chronic constrictive pericarditis were enrolled who were documented to have tuberculous pericardial effusion in the past. The patients who had other causes of pericardial effusion were excluded such as deranged thyroid function, connective tissue disorder or malignancy found elsewhere in the body or positive malignant cells in pericardial fluid and in whom no evidence of tuberculosis was detected for 6 months after initial presentation.

\section{Result:}

During the study period, 53 patients were included of whom $62 \%$ were male and $38 \%$ were female patients. The ages ranged from 6-71years $(42 \pm 19.5)$. (Figure 1) The most common manifestation seen was large pericardial effusion (30\%) followed by CCP (22.6\%), 19\% presented in cardiac tamponade, others were pyopericardium, perimyocarditis and acute pericarditis. (Figure 2)

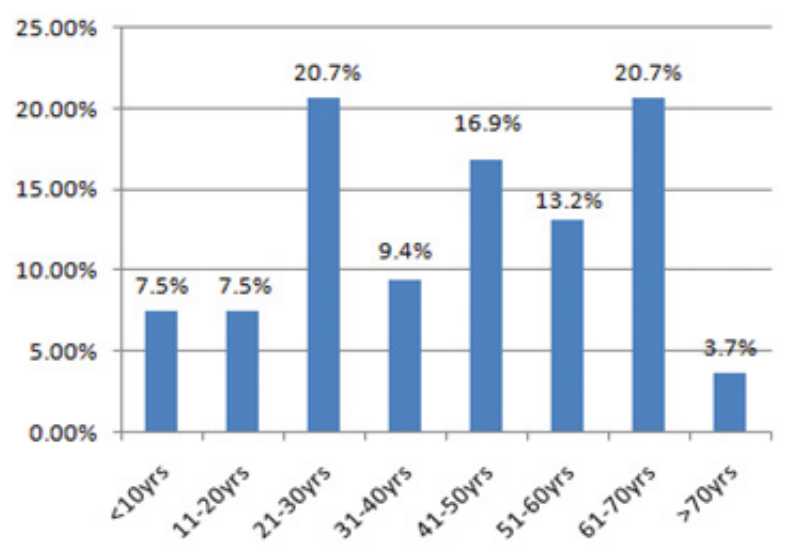

Figure 1: Age of patients presenting with tuberculos pericarditis
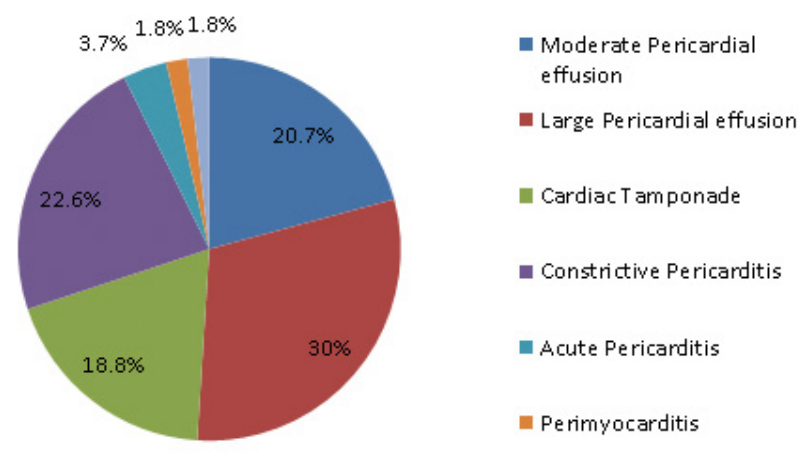

Figure 2: Different cardiac manifestations of tuberculosis

History of contact with tuberculosis was present in 11 patients $(20 \%)$. The patients with pericardial effusion, pericarditis and pyopericardium presented with symptoms such as fever, dyspnea, and chest pain usually insidious in onset. (Table 1) The patients with constrictive pericarditis presented with abdominal distension, legs swelling and dyspnea of prolonged duration. The total peripheral leucocyte count ranged from 3.1-14 x 10\%/litre with mean of $7.8 \times 109 /$ 
liter. Echocardiography of thirty-one patients $(60 \%)$ had fibrin strands crisscrossing the pericardial space. Ten patients $(20 \%)$ had associated pleural effusion and one patient had loculated empyema thoracis. Pericardiocentesis was done in 20 patients with large pericardial effusion who had or developed cardiac tamponade. ADA of the pleural fluid or pericardial fluid whichever was available supported for tubercular effusion. The ADA was positive in $75 \%$ of the cases when pericardial fluid was analyzed whereas it was positive in only $40 \%$ of the cases when pleural fluid was analyzed. The fluids had lymphocytic predominance in most i.e. $81 \%$ in pericardial fluid and $90 \%$ in pleural fluid. The Absolute Lymphocyte Count (ALC) of pericardial fluid was high with mean of 2855 cells $/ \mathrm{mm}^{3}$. Pericardial biopsy was taken when pericardial window was made in 2 patients with large pericardial effusion but showed non-specific chronic inflammation. The tuberculin skin test was done in all patients and measured $2-26 \mathrm{~mm}$ and it was positive $(\geq 10 \mathrm{~mm})$ in $18 \%$ of the cases where it supported the diagnosis when the body fluid wasn't available for analysis specially with small to moderate pericardial effusion.

\begin{tabular}{|lc|}
\hline Table 1: Baseline characteristics & \\
\hline Features & Number of Patients \\
\hline Duration (in days) & $12-120$ \\
\hline Fever & 16 \\
\hline Cough & 15 \\
\hline Dyspnea & 38 \\
\hline Chest pain & 7 \\
\hline Abdominal distension & 13 \\
\hline
\end{tabular}

The patients were given anti tubercular treatment (ATT) including isoniazid, rifampicin, pyrazinamide and ethambutol as well as prednisolone as per national guideline who were diagnostically proven and those who didn't have confirmatory evidence of tuberculosis. The patients were followed up till the completion of ATT with echocardiography. The pericardial effusion had resolved in most patients and was thus included in this study. However, two of the patients with cardiac tamponade developed CCP on follow up after 3 and 4 months. The patients with CCP initially diagnosed at presentation had undergone pericardiectomy after 4-6 weeks of antitubercular treatment. There was one case with seropositivity for Hepatitis B and one positive for Human Immunodeficiency Virus (HIV). Two patients died of whom one was case of CCP due to decompensated heart failure and other case was HIV seropositive with chronic kidney disease in cardiac tamponade whose pericardiocentesis was done but the patient succumbed due to cardiac arrest secondary to refractory hyperkalemia.

\section{Discussion:}

The awareness of tuberculosis as the possible cause of a pericardial effusion often depends on the frequency with which tuberculosis is encountered in practice. ${ }^{6}$ Tuberculous pericarditis is common in all age groups with varying features like fever, dyspnea, chest pain, abdominal distension and leg swelling. Reuter et al studied 233 cases of pericardial effusion that showed that fever, night sweats, weight loss, and peripheral blood leukocyte count $\left(<10 \times 10^{\circ} / \mathrm{l}\right)$ were independently predictive of tuberculous pericarditis. Pericardial fluid ADA $\geq 40 \mathrm{U} / 1$ had $87 \%$ sensitivity and $89 \%$ specificity. $^{7}$ Our study also showed the usefulness of basic diagnostic tools to assist clinical decision-making especially in poor resource countries like ours for instance, raised pericardial fluid $\mathrm{ADA}$ has been the major evidence for diagnosing tuberculous pericarditis and similar to this study the mean peripheral blood leukocyte count was $7.8 \times 10^{9} /$ liter. ADA is a valuable marker with both high sensitivity and specificity in the diagnosis of tuberculous pericarditis but with relative sensitivity and specificity for the diagnosis of tuberculous pleurisy. ${ }^{8,9}$ In a metaanalysis of 11 studies, 938 subjects were included which showed the sensitivity of ADA as 0.90 and specificity as $0.86 .{ }^{8} \mathrm{In}$ our study also ADA remained as a valuable marker for tubercular pericardial effusion than tubercular pleural effusion.

Mutyaba et al had mentioned from his review article that was retrieved from sub-Saharan Africa and Asia that among the 3 predominant clinical manifestations of tuberculous heart disease, the most frequent is TB pericarditis followed by myocardial $\mathrm{TB}$ with or without aneurysm formation, and TB aortitis with or without mycotic aneurysms and pseudoaneurysms involving the aortic valve and/or sinuses of Valsalva. In the developing world, $40 \%$ to $70 \%$ of large pericardial effusions are tuberculous in origin whereas in the developed world, less than $4 \%$ of cases are tuberculous. ${ }^{10}$ Tuberculous myocarditis showed to be a rare manifestation of cardiovascular TB with an occurrence rate of $0.14 \%$ in more than 13,000 autopsies performed over 27 years by Rose and colleagues. ${ }^{11}$ Tuberculous infection of the aorta seemed to be an exceedingly rare manifestation of TB with an occurrence rate of $0.004 \%$ in 22,792 postmortems examinations over 50 years. ${ }^{10}$ In our study also the most common cardiac manifestation of tuberculosis seen was tuberculous pericarditis in the form of large pericardial effusion (30\%) followed by CCP (22.6\%), 19\% presented in cardiac tamponade, others were pyopericardium, perimyocarditis and acute pericarditis. However the rarer manifestations of tuberculosis as found in other studies were not found in this study.

Tuberculous pericarditis is the commonest manifestation of TB in the cardiovascular system, and pericardial constriction is dreaded sequelae, occurring in approximately $8 \%$ of affected individuals despite effective anti-TB therapy. ${ }^{12} \mathrm{~TB}$ is, by far, the most common cause of pericardial constriction in the developing world, accounting for about $40-90 \%$ of cases seen in different series. ${ }^{13}$ By contrast, less than $5 \%$ of cases of pericardial constriction in the developed world are attributable to TB. ${ }^{14}$

Cherian et al in his review article has suggested the criteria for diagnosis of tuberculous etiology of pericardial effusion: Invasive criteria was culture of Mycobacterium tuberculosis from pericardial fluid or tissue, pericardial tuberculous granuloma with acid-fast bacilli, pericardial tuberculous granuloma with positive tuberculin skin test, tuberculous granuloma in scalene node or peripheral lymph node or pleura with positive tuberculin test. Non-invasive criteria was active tuberculosis elsewhere in the body and response to specific antitubercular therapy. ${ }^{15}$ In our study, the noninvasive criteria and indirect evidences typically became more practical and valuable.

Echocardiography is the definitive investigation for pericardial effusion and tamponade, particularly valuable for distinguishing effusion from subacute constriction. ${ }^{16,17}$ Cherian et al did a study where the patients with tubercular pericardial effusion pericardial deposits and strands in pericardial space on echocardiography and $31 \%$ of patients had positive culture for M. tuberculosis from pericardial fluid or biopsy. ${ }^{18}$ Through echocardiography we found fibrinous strands and pericardial thickening suggesting tubercular origin however, our study couldn't isolate the organism by culture.

A strongly positive tuberculin skin test result may increase the suspicion of tuberculous pericarditis, but a negative test does not exclude this diagnosis. ${ }^{7}$ Only 13 patients in our study had positive tuberculin test positive. Mishra et al found that fluid PCR for Mycobacterium tuberculosis was positive in $74 \%$ of tuberculous effusions. The mean fluid ADA and Absolute Lymphocyte Count (ALC) values were significantly higher in tuberculous effusions than in non-tuberculous effusions. The sensitivity and specificity 
of PCR, ADA (>38 IU/l) and ALC (>275/mm3) were $74 \%$ and $88 \%, 81 \%$ and $75 \%$, and $90 \%$ and $83 \%$, respectively, in diagnosing tuberculous effusions. ${ }^{19}$ In our study, the ADA level and absolute lymphocyte count of tuberculous pericardial effusions were significant for the diagnosis but PCR was not done as it wasn't available within the center. Desai et al did a retrospective survey of 100 black patients with presumed tuberculous pericarditis showed 82 with pericardial effusion while 18 had constrictive pericarditis. The mortality rate was $17 \%$. Of 82 with pericardial effusion, 15 developed constrictive pericarditis within 4 months, 16 died of cardiac tamponade..$^{20}$ Our study found that most common cardiac manifestation of tuberculosis was pericardial effusion followed by CCP and showed that despite antitubercular treatment pericardial effusion may progress to constriction.

In the developed countries, the HIV seropositivity is mainly the cause for TB pericarditis but in our study only one patient was seropositive for HIV. In country like ours, the reason is still the low economic condition so that even the younger patients suffer chronic constrictive pericarditis.

The potential benefit of corticosteroids is to quicken resolution of symptoms and decrease reaccumulation of fluid but didn't significantly affect the risk of death or progression to CCP. ${ }^{21}$ After pericardiectomy, patients of CCP had good outcome except one patient who died immediately after the procedure. The favourable outcomes of antitubercular chemotherapy and timely pericardiectomy was seen in a study done by Yang CC et al .22,23

\section{Conclusion:}

Among all the extrapulmonary manifestations of $\mathrm{TB}$, involvement of the heart is second only to central nervous system TB in terms of its devastating morbidity and mortality. Tuberculosis leads to any of the four clinical manifestations of tubercular pericarditis i.e acute pericarditis, effusive pericarditis, myopericarditis and CCP as well as myocarditis and aortitis. However it commonly manifests as pericarditis in developing countries like Nepal regardless of any age or gender. Since there is lack of improved techniques for recovery of $M$. tuberculosis such as PCR technology, pericardial IFN-g, biopsy, culture, we are still dependent only on 2D echo, ADA levels of pericardial fluid, supportive evidence of tuberculosis elsewhere in the body and clinical history of therapeutic response after anti tuberculosis chemotherapy. Had we got such sophisticated investigations done easily, the incidence of tuberculous pericarditis would have been assessed presumably better since early diagnosis and institution of appropriate therapy with antitubercular drugs are critical to prevent mortality.

\section{References:}

1. American Thoracic Society. Diagnostic Standards and Classification of Tuberculosis in Adults and Children. Am J Respir Crit Care Med. 2000;161:1376-1395. https://doi. org/10.1164/ajrccm.161.4.16141

2. K Wanjari, V Baradkar, M Mathur, S Kumar: A case of tuberculous pericardial effusion: Indian Journal of Medical Microbiology Year. 2009; 27 (1): 75-77

3. Bongani M. Mayosi, Lesley J. Burgess, Anton F. Doubell. Tuberculous Pericarditis: Circulation. 2005;112:3608-3616

4. Milena Adina Man et al. Tuberculous constrictive pericarditis complications. Rom J Morphol Embryol 2016; 57(1):237242

5. Shweta Rana et al. Tuberculous Pericarditis on autopsy: A case report Eur J Forensic Sci. 2015; 2(2): 27-29

6. Cherian G, Uthaman B, Habashy AG, Salama AL, George S. Large pericardial effusion: the differentiation of tuberculous

Cite this article as: Smriti Shakya, Sunil Chandra Jha. Cardiac manifestations of tuberculosis in a tertiary care center of Nepal. Nepalese Heart Journal 2018; 15(1) 35-38: http://dx.doi.org/10.3126/njh.v15i1.19714 from chronic idiopathic effusion. J Assoc Physicians India. 2003 Sep; 51:880-3.

7. Reuter H, Burgess L, van Vuuren W, Doubell A. "Diagnosing tuberculous pericarditis". Q J Med(2006);99 (12): 827-39. https://doi.org/10.1093/qjmed/hcl123

8. Xie DL, Cheng B, Sheng Y, Jin J.Diagnostic accuracy of adenosine deaminase for tuberculous pericarditis: a metaanalysis. Eur Rev Med Pharmacol Sci. 2015 Nov;19(22):44118 .

9. Qiu-Li Liang, Huan-Zhong Shi, Ke Wang, Shou-Ming Qin, Xue-Jun Qin. Diagnostic accuracy of adenosine deaminase in tuberculous pleurisy: A meta-analysis.Respiratory Medicine 2008; 102: 744-754. https://doi.org/10.1016/j. rmed.2007.12.007

10. Arthur K. Mutyaba, Mpiko Ntsekhe. Tuberculosis and the Heart. Cardiol Clin 35 2017; 135-144. https://doi. org/10.1016/j.ccl.2016.08.007

11. Rose AG. Cardiac tuberculosis. A study of 19 patients. Arch Pathol Lab Med 1987;111(5):422-6.

12. Mayosi BM, Ntsekhe M, Bosch J et al. Prednisolone and Mycobacterium indicus pranii in Tuberculous Pericarditis. New England Journal of Medicine 2014;371:1121-1130. https://doi.org/10.1056/NEJMoa1407380

13. Mutyaba AK, Balkaran S, Cloete $\mathrm{R}$ et al. Constrictive pericarditis requiring pericardiectomy at Groote Schuur Hospital, Cape Town, South Africa: Causes and perioperative outcomes in the HIV era (1990-2012). The Journal of Thoracic and Cardiovascular Surgery 2014;148:3058-3065. e1. https://doi.org/10.1016/j.jtcvs.2014.07.065

14. Imazio M. Constrictive Pericarditis. Myopericardial Diseases: Diagnosis and Management. Cham: Springer International Publishing, 2016:131-140. https://doi.org/10.1007/978-3319-27156-9

15. G Cherian et al. Diagnosis of tuberculous aetiology in pericardial effusions. Postgrad Med J 2004;80:262-266. https://doi.org/10.1136/pgmj.2003.013664

16. Schepers GWH. Tuberculous pericariditis. Am J Cardiol 1962;9:248-76. https://doi.org/10.1016/00029149(62)90044-9

17. Hageman JH, D'Esopo ND, Glenn WWL. Tuberculosis of the pericardium: a long-term analysis of forty-four proved cases. N Engl J Med 1964; 270: 327-332. https://doi. org/10.1056/NEJM196402132700702

18. Cherian G, Uthaman B, Habashy AG, Salama AL, George S. Large pericardial effusion: the differentiation of tuberculous from chronic idiopathic effusion. J Assoc Physicians India. 2003 Sep;51:880-3.

19. O P Mishra, R Kumar, Z Ali, R Prasad, G Nath. Evaluation of polymerase chain reaction and adenosine deaminase assay for the diagnosis of tuberculous effusions in children. Arch Dis Child 2006;91:985-989. https://doi.org/10.1136/ adc. 2005.079160

20. Desai HN et al. Tuberculous pericarditis. A review of 100 cases. S Afr Med J. 1979; 55(22):877

21. Strang JI, Kakaza HH, Gibson DG, et al. Controlled trial of prednisolone as adjuvant in treatment of tuberculous constrictive pericarditis in Transkei. Lancet 1987; 2(8573): 1418-22. https://doi.org/10.1016/S0140-6736(87)91127-5

22. Yang CC, Lee MH, Liu JW, Leu HS. Diagnosis of tuberculous pericarditis and treatment without corticosteroids at a tertiary teaching hospital in Taiwan: a 14-year experience. J Microbiol Immunol Infect. $2005 \mathrm{Feb} ; 38(1): 47-52$.

23. McCaughen BC, Hartzell V, Schaff HV, Piehler JM, Danielson GK, Orsulak TA, et al. Early and late results of pericardiectomy for constrictive pericarditis. J Thorac Cardiovasc Surg. 1985;89:340-50. 